\title{
BEAK-SHAPED GRAIN 1/TRIANGULAR HULL 1, a DUF640 gene, is associated with grain shape, size and weight in rice
}

\author{
YAN DaWei ${ }^{1 \dagger}$, ZHOU Ya $^{2 \dagger}$, YE ShengHai ${ }^{2}$, ZENG LongJun $^{1}$, ZHANG XiaoMing $^{2 *}$ \\ \& $\mathrm{HE} \mathrm{ZuHua}^{1 *}$

\begin{abstract}
${ }^{1}$ National Key Laboratory of Plant Molecular Genetics, Institute of Plant Physiology and Ecology, Shanghai Institutes for Biological Sciences, Chinese Academy of Sciences, Shanghai 200032, China;

${ }^{2}$ State Key Laboratory Breeding Base for Zhejiang Sustainable Pest and Disease Control, Institute of Crop and Nuclear Technology Utiliza-
\end{abstract} \\ tion, Zhejiang Academy of Agricultural Sciences, Hangzhou 310021, China
}

Received October 17, 2012; accepted December 21, 2012

\begin{abstract}
Grain shape and size both determine grain weight and therefore crop yield. However, the molecular mechanisms controlling grain shape and size are still largely unknown. Here, we isolated a rice mutant, beak-shaped grain1 (bsg1), which produced beak-shaped grains of decreased width, thickness and weight with a loosely interlocked lemma and palea that were unable to close tightly. Starch granules were also irregularly packaged in the $b s g 1$ grains. Consistent with the lemma and palea shapes, the outer parenchyma cell layers of these $b s g l$ tissues developed fewer cells with decreased size. Map-based cloning revealed that BSG1 encoded a DUF640 domain protein, TRIANGULAR HULL 1, of unknown function. Quantitative PCR and GUS fusion reporter assays showed that BSG1 was expressed mainly in the young panicle and elongating stem. The BSG1 mutation affected the expression of genes potentially involved in the cell cycle and $G W 2$, an important regulator of grain size in rice. Our results suggest that $B S G l$ determines grain shape and size probably by modifying cell division and expansion in the grain hull.
\end{abstract}

cell division and expansion, DUF640 protein, gene expression, grain size, lemma and palea

Citation: Yan D W, Zhou Y, Ye S H, et al. BEAK-SHAPED GRAIN 1/TRIANGULAR HULL 1, a DUF640 gene, is associated with grain shape, size and weight in rice. Sci China Life Sci, 2013, 56: 275-283, doi: 10.1007/s11427-013-4449-5

As a staple food crop worldwide, rice has been a major target of research and breeding. Grain weight, number of grains per panicle and number of panicles per plant are known as the crucial factors of grain yield in rice. In recent years, a number of genes related to these factors have been recognized [1-10]. Several of these genes, including $G W 2$, $G S 5$ and $G S 3$, control grain weight by regulating the shape and size of grains. GW2 encodes a RING-type E3 ubiquitin ligase and was shown to negatively regulate cell division [5]. $G S 5$, a positive regulator controlling grain width and weight,

$†$ Contributed equally to this work

*Corresponding author (email: xmzhang@mail.hz.zj.cn; zhhe@sibs.ac.cn) encodes a putative serine carboxypeptidase [11]. In addition, $G S 3$, which encodes a putative transmembrane protein, functions as a major QTL for grain length and weight $[4,12]$.

In a typical rice spikelet, the grain hull, consisting of a lemma and palea, determines the grain shape and size. The leafy hull sterilel (lhsl) mutant, with a homeotic mutation in OSMADS1, exhibits leaf-like paleas, lemmas and lodicules [13]. RETARDED PALEAI (REPI) regulates palea identity and development in rice [14], and the cells in repl paleas are smaller and less differentiated than those in the wild-type. REPI is regulated by the upstream gene DEPRESSED PALEAl (DPl), an AT-hook gene required 
for normal palea formation [15]. In the rice open beak (opb) mutant, the lemma bends towards the palea and the lateral growth of the lemma and palea is suppressed such that they are unable to close [16].

The basic cell cycle machinery that regulates cell division for growth and development is highly conserved in eukaryotes [17-19]. Different cyclin-dependent kinase (CDKs) complexes phosphorylate different substrates at the $\mathrm{G}_{1}$-to-S and $\mathrm{G}_{2}$-to-M transition points to induce DNA replication and mitosis to control the cell cycle. For example, CDKA is essential at both the $\mathrm{G}_{1}$-to- $\mathrm{S}$ and $\mathrm{G}_{2}$-to-M transitions of the cell cycle in plants $[20,21]$. $C D K B 1$ is expressed during the $\mathrm{S}$ to $\mathrm{M}$ phases while $C D K B 2$ is mainly expressed in the $G_{2}$ to $M$ phases [22-24]. CDK-activating kinases (CAKs) and cyclin-dependent kinase inhibitors (CKIs) modulate the level of CDK activity together to control cell cycle progression [25-28].

In this study, we isolated two allelic mutants, beakshaped grain1-1 (bsg1-1) and bsg1-2, whose palea and lemma develop incompletely and whose grain width, thickness and weight are all significantly reduced. BSGl encodes a protein containing a DUF640 domain of unknown function. Our results show that $B S G 1$ regulates the expression of cell cycle genes and floral homeotic genes in the grain hull. This work demonstrates that BSG1 controls grain hull development and provides further evidence for the functional exploration of DUF640 proteins.

\section{Materials and methods}

\subsection{Plant materials and mutants isolation}

The $b s g 1-1$ and $b s g 1-2$ mutants were identified from a ${ }^{60} \mathrm{Co}$ $\gamma$-ray-mutagenized population of cultivar Zhejing22 (Oryza sativa ssp. japonica). The mutants were crossed with Zhejing22 for genetic analysis and with 93-11 (ssp. indica) for genetic mapping. All of the parents, F1 hybrids and corresponding F2 individuals were grown in paddy fields at Shanghai and Hainan under natural conditions.

\subsection{Phenotyping of grain traits}

Fully filled and air-dried grains were used for measurements. Thirty grains of each genotype were measured for grain length and width, and 3000 grains were weighed for 1000 -grain weight. The cell lengths in the hulls were measured using the ImageJ software.

\subsection{Map-based cloning}

Mutant individuals showing the $b s g l$ phenotype in the F2 population derived from $b s g 1-1$ and 93-11 were used for map-based cloning. An initial screening for molecular markers linked to $B S G 1$ was performed using markers from publicly available rice databases, including Gramene (http:// www.gramene.org) and the Rice Genomic Research Program (http://rgp.dna.affrc.go.jp/). Fine mapping was performed using new markers developed from genomic polymorphisms between 93-11 (http://rise.genomics.org.cn/rice/ index2.jsp) and Nipponbare (ssp.japonica, http://rgp.dna. affrc.go.jp/cgi-bin/statusdb/status.pl). The main markers are listed in Table S1.

\subsection{Histological analysis}

Young spikelets before heading were fixed in FAA $(70 \%$ ethanol, 5\% glacial acetic acid, 3.7\% formaldehyde), embedded in Epon 812 resin, cut into $2-\mu \mathrm{m}$ sections and stained with toluidine blue. Sections were examined under a microscope (BX51, Olympus, Tokyo, Japan) and photographed. For scanning electron microscopy (SEM), the samples were fixed overnight at $4^{\circ} \mathrm{C}$ in FAA and dehydrated in a graded ethanol series. The samples were then critical-point dried in liquid carbon dioxide and coated with gold, followed by visualization with a scanning electron microscope (JSM6360LV, JEOL, Tokyo, Japan).

\subsection{GUS reporter assay}

The GUS coding sequence was released by digesting the vector pBI101 with Hind III and EcoR I, ligated with a 1950-bp promoter fragment of the BSG1 gene, and inserted into the binary vector pCAMBIA1300 to generate the fusion reporter plasmid $\mathrm{p} B S G 1-G U S$, which was then transformed into $O$. sativa cv. Nipponbare by an Agrobacterium tumefaciens-mediated method [29]. Different tissues and organs of the transgenic plants were collected for histochemical assays of GUS activity.

\subsection{RNA isolation and expression assay}

Total RNA was isolated from different tissues using TRIzol reagent according to the manufacturer's protocol (Invitrogen, Carlsbad, CA, USA) and treated with DNase I using the DNA-free kit (Ambion, Austin, TX, USA). Real-time PCR (quantitative PCR) was performed in triplicate using the SYBR Premix Ex Taq kit (TaKaRa, Otsu, Japan) and the Mastercycler ep realplex detection system (Eppendorf, Hamburg, Germany). The amplification program was as follows: $95^{\circ} \mathrm{C}$ for $3 \mathrm{~min}$, followed by 40 cycles at $95^{\circ} \mathrm{C}$ for $20 \mathrm{~s}$, and $60^{\circ} \mathrm{C}$ for $1 \mathrm{~min}$. ACTIN2 was amplified as an internal control for loading normalization. Relative transcript levels were obtained using the $2^{-\Delta \Delta C_{t}}$ quantification method [30]. All primers are listed in Table S1.

\section{Results}

\subsection{Grain phenotypes of the bsg1-1 mutant}

The bsg 1-1 mutant was isolated from the M2 population of 
a Zhejing22 mutant pool treated with ${ }^{60} \mathrm{Co} \gamma$-rays. There was no obvious difference between the mutant and the wild-type during the vegetative stage. In adult plants, bsg 1-1 showed no significant differences in architecture traits such as plant height and tiller number, compared with the wild-type. However, the hull of the mature grain in bsg 1-1 was bend and not completely closed, making it look like a beak (Figure 1A). Phenotypic analysis showed that grain width and thickness in $b s g 1-1$ were reduced by $25.1 \%$ and $17.1 \%$, respectively, compared with the wild-type (Figure $1 \mathrm{~A}$ and $\mathrm{D})$, whereas grain length did not change significantly (Figure 1B). The conical seeds of $b s g 1-1$ had dark brown coats and were also smaller than the seeds of the wild-type (Figure 1A).

To investigate the influence of $B S G 1$ on grain weight, we compared $b s g 1-1$ with the wild-type and observed a significant decrease (50.2\%) in 1000-grain weight in bsg 1-1 (Figure 2A). The bsgl-1 grain also showed markedly abnormal development and irregular packaging of starch granules (Figure 2B). These results together indicated that BSG1 affected not only grain shape but also grain weight.

\subsection{Abnormality of lodicules in bsg 1-1}

Besides the lemma and palea, the lodicules in $b s g 1-1$ were also abnormal compared with those of the wild-type (Figure $3 \mathrm{~A}, \mathrm{~B}, \mathrm{D}$, and E). The upper regions of the lodicules were elongated and a few awn-like protuberances were formed on their top in bsgl-1 (Figure 3A and B). SEM images showed that the cells in the elongated parts of the lodicules differed to those of wild-type lodicules; they lacked the striation of the wild-type epidermal cells (Figure 3C and F). This implied that the differentiation of specific cells in the lodicules might be changed in bsgl-1 and suggested that BSGl was involved in lodicule development.

\subsection{Map-based cloning of the BSG1 gene}

The bsg1-1 mutant was crossed with the wild-type (Zhejing22) and all F1 hybrids displayed the wild-type phenotype. In the F2 population, the segregation ratio of wild-type to bsg 1-1 plants showed typical Mendelian segregation $\left(3: 1, \chi^{2}=1.59<\chi_{0.05,1}^{2}=3.84\right)$. This result indicated that the mutant phenotype was controlled by a single recessive gene. An F2 population with 560 individuals displaying the bsgl mutant phenotype from a cross between bsgl-1 and 93-11 was used for mapping. BSGl was finally narrowed down to a region of 194-kb between markers J59 and J95, which contained partial sequences of three BAC clones: AP004081, AP005303 and AP005691 (Figure 4A).

Based on the available sequence annotation database (http://rapdblegacy.dna.affrc.go.jp/), 28 predicted genes were located in the 194-kb target region. We compared the genome sequences of these candidate genes in bsgl-1 and Zhejing22 and a 5-bp deletion in the genomic sequence of the predicted gene $0 s 02 g 0811000$ was identified in $b s g 1-1$ (Figure 4B) and verified by restriction enzyme digestion patterns. According to the annotation database, the fulllength cDNA of Os02g0811000 is $1587 \mathrm{bp}$, containing a 747-bp coding sequence that encodes a putative protein with an unknown-function domain called DUF640 (Figure 4B).

Besides $b s g 1-1$, we identified an additional beak-shaped
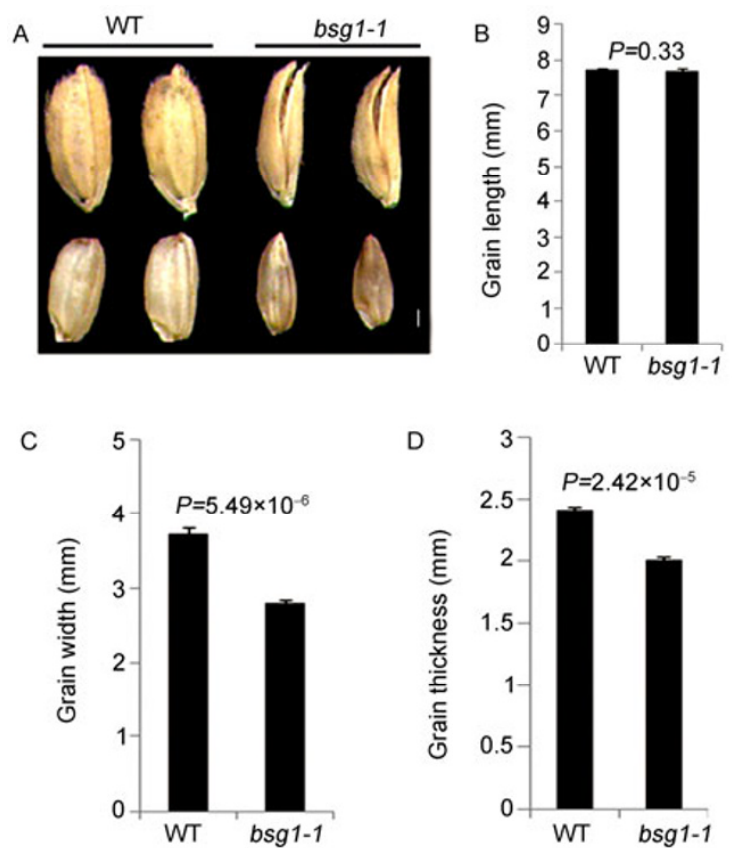

Figure 1 Characterization of the $b s g 1-1$ mutant. A, Grain phenotypes of the wild-type and bsgl-1. Scale bar, $1 \mathrm{~mm}$. B-D, Comparisons of grain length (B), grain width (C) and grain thickness (D) in the wild-type and bsg1-1. Data are means $\pm \mathrm{SD}(\mathrm{N}=30)$. The $P$-values were generated by $t$-tests (in color in the electronic version).

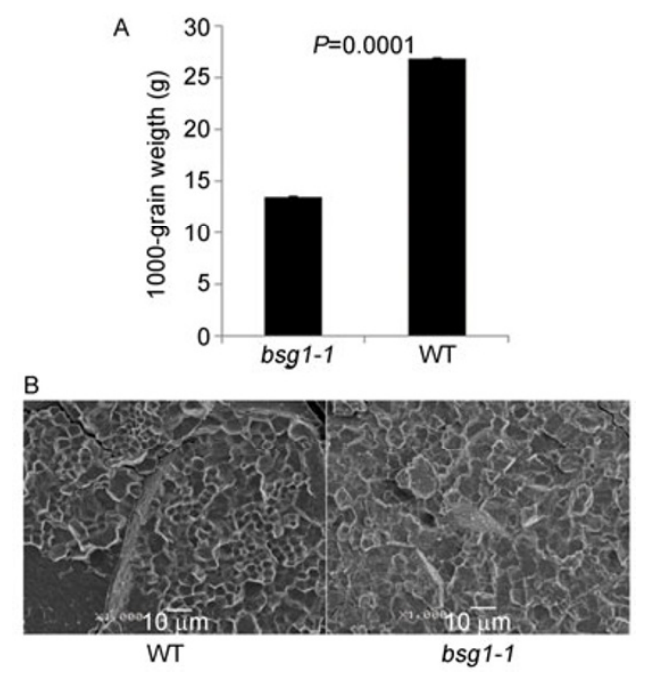

Figure 2 Grain weight and starch granules of the wild-type and $b \operatorname{sg} 1-1$. A, 1000-grain weight in the wild-type and $b s g 1-1$. The $P$-value was generated with a $t$-test. B, SEM images of starch granules in wild-type and bsg1-1 grains. 

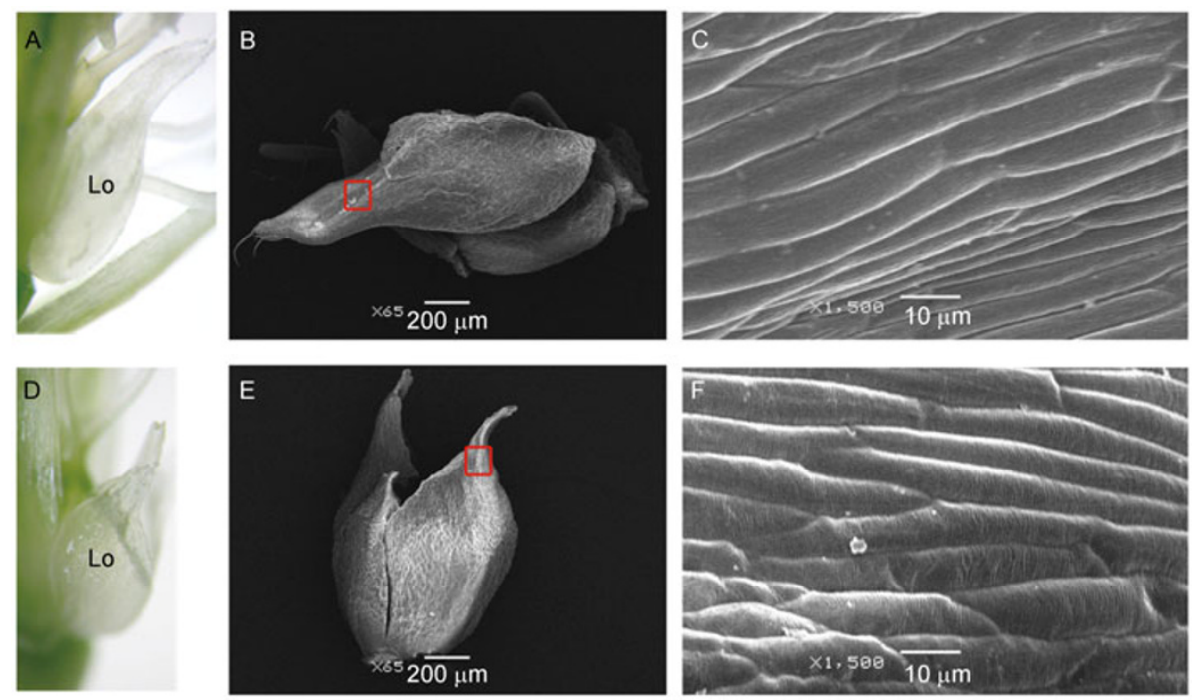

Figure 3 Lodicule phenotype in $b s g 1-1$. A and D, Elongated lodicules of $b s g 1-1$ (A) and normal lodicules of the wild-type (D). Lo, lodicule. B and E, SEM images of bsg1-1 (B) and wild-type (E) lodicules. C and F, Magnified SEM images of the apical cells of lodicules in bsg1-1 (C) and the wild-type (F) (red boxed regions in $\mathrm{B}$ and $\mathrm{E}$ )(in color in the electronic version).
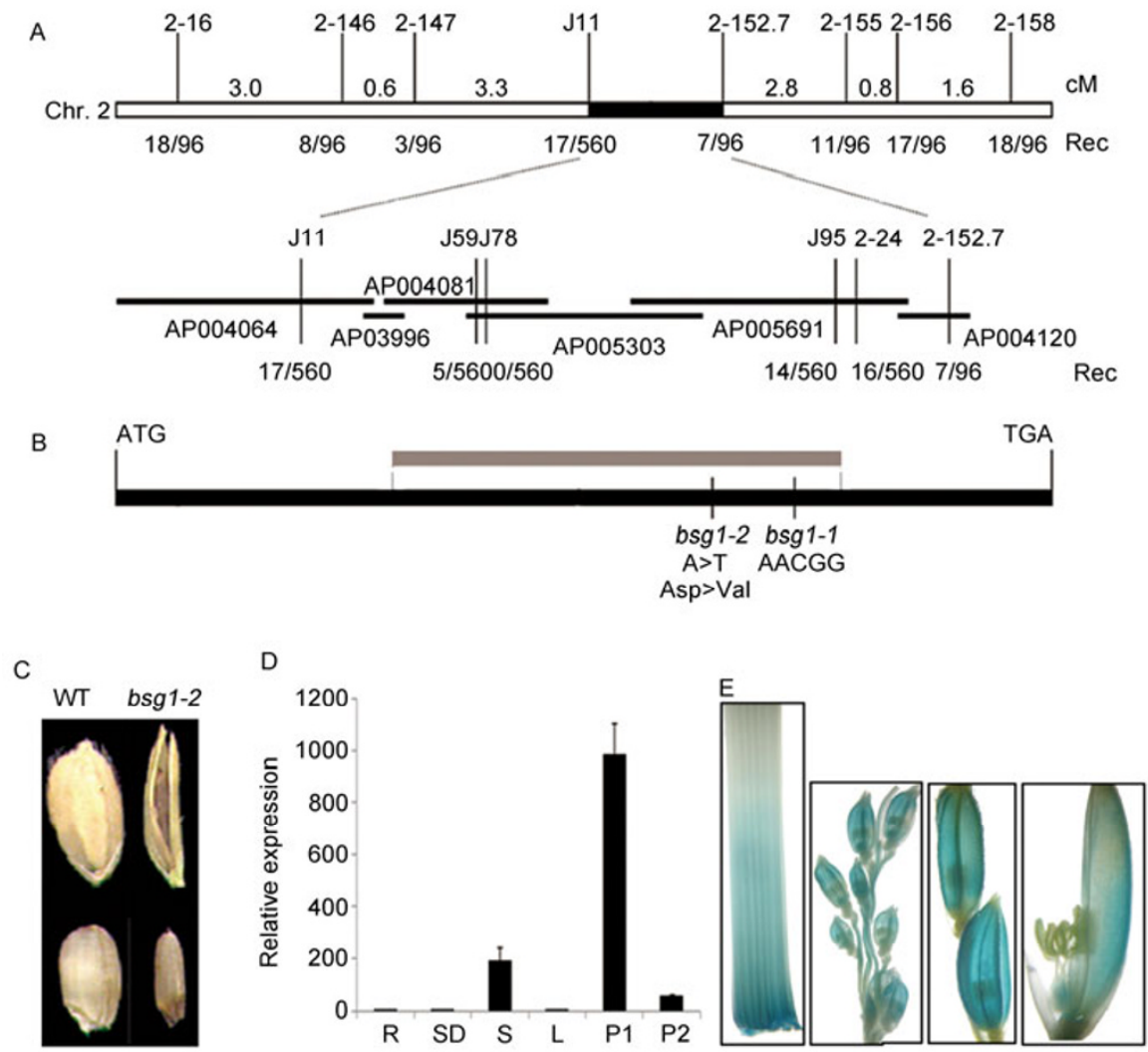

Figure 4 Map-based cloning of BSG1. A, Location of BSG1 on rice chromosome 2 between markers J59 and J95. This region contains three partial BAC sequences. Chr, chromosome; cM, centimorgan; Rec, recombinant. B, Coding sequence schematic of the candidate gene $(O s 02 g 0811000)$ and mutation sites in bsg1-1 and bsg1-2. The gray box shows the sequence segment that encodes the DUF640 domain. C, Grain phenotypes of the wild-type and bsg1-2. D, $B S G 1$ expressions in different tissues, as determined by real-time PCR. R, root; SD, seedling; S, stem; L, leaf; P1, young panicle (inflorescence length from $0.1-2 \mathrm{~cm}$ ); P2, young panicle (inflorescence length from 8-10 cm). E, GUS reporter activity in stems, hulls of young spikelets $(2 \mathrm{~cm})$, hulls of developed spikelets $(10 \mathrm{~cm})$ and lodicules of transgenic plants, from left to right (in color in the electronic version).

grain mutant from the same M2 population, $b s g 1-2$ (Figure 4C), which was also controlled by a single recessive gene.
Sequencing revealed a transition from $\mathrm{A}$ to $\mathrm{T}$ in the exon of the same Os02g0811000 gene, resulting in a change of 
amino acid from Asp to Val (Figure 4B). The deletion in bsg 1-1 and amino acid transition in bsg 1-2 both occurred in the DUF640 coding sequence (Figure 4B). Recently, another mutant, triangular hull1-1 (th1-1) in the same gene was reported with a similar phenotype [31]. Taken together, these results demonstrated that the $0 s 02 g 0811000$ gene was responsible for the phenotype of $b$ s $1 /$ th $1-1$.

Real-time PCR analysis of the BSGl expression pattern showed that transcripts of BSGl were accumulated most strongly in the young panicles (inflorescence length from $0.1-2 \mathrm{~cm}$ ), followed by stems and older panicles (inflorescence length from 8-10 cm) (Figure 4D). Almost no expression of $B S G 1$ was detected in roots, seedlings and mature leaves. These results were consistent with the expression patterns detected by the $B S G 1:: G U S$ fusion reporter in transgenic plants (Figure 4E).

\subsection{Histological characterization of the lemma and palea}

To study the histological phenotype of the hull, we examined cross-sections of the central part of the lemma and palea of young spikelets. The floret of $b s g 1-1$ was open and lacked interlocking between the lemma and palea (Figure 5C), compared with the wild-type that was hooked closely (Figure 5A). The outer parenchyma cell layers of the lemma and palea (Figure 5B and D) in bsgl-l were both shorter and contained fewer cells than the wild-type (Figure 5E and F). These features demonstrated that the reduced width of the $b s g 1-1$ spikelet hull resulted mainly from the decrease in cell number and size. Moreover, in wild-type plants, the lateral margin tissue of the palea (MTP), which differed from the rest of palea, was membranous with a smooth surface and lacked epicuticular and silicified thickening (Figure 5B). However, the $b s g 1-1$ palea lacked distinctive marginal tissue structure and had more parenchyma cell layers than the wild-type (Figure 5D). These morphological characteristics suggested that the cell identity of the lateral margin tissue of the palea was lost and that the cell specification was disturbed in $b s g l-1$.

\subsection{Effect of BSG1 on the expression of cell-cycle genes}

Since cell identity and cell specification were disturbed in bsg1-1, we investigated whether cell-cycle genes were regulated by $B S G 1$. We analyzed the expression levels of 25 rice genes, identified by querying sequences of previously published core cell cycle regulators [11], in the hulls of young panicles in the $b s g l-1$ mutant and the wild-type (Figure 6A). Among them, the transcript levels of 11 genes were greatly reduced in $b s g 1-1$. Moreover, the expression level of $C D C 20$, an activator of the anaphase-promoting complex (APC) for mitosis exit via destruction of cyclin subunits [17,32], was elevated in $b s g 1-1$. Therefore, it appears that $B S G 1$ likely acts as a positive regulator of cell
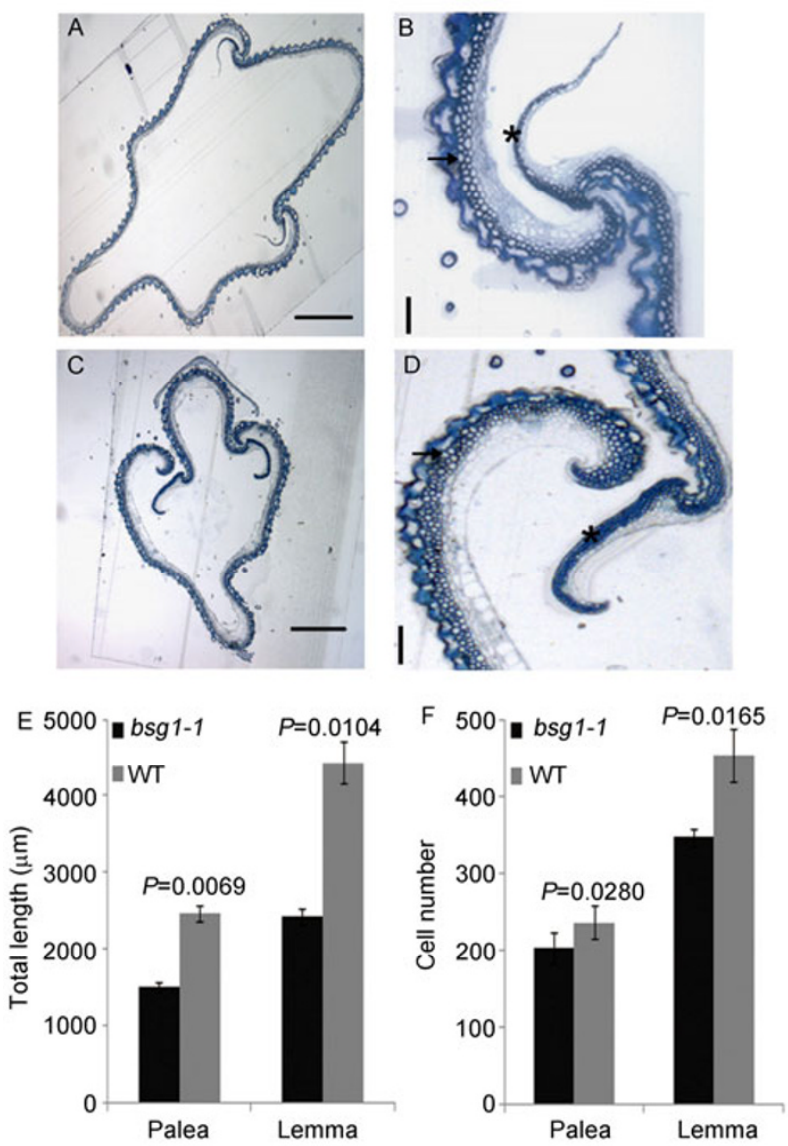

Figure 5 Histological analysis of spikelet hulls in the wild-type and bsg1-1. A and C, Cross sections of wild-type (A) and bsg1-1 (C) grain hulls. Scale bar, $1 \mathrm{~mm}$. B and D, Cross sections of wild-type (B) and bsg1-1 (D) palea edges. Arrows show the outer parenchymal cell layers. Asterisks indicate the margin tissue of the palea. Scale bar, $100 \mu \mathrm{m}$. E and $\mathrm{F}$, Comparison of total length (E) and cell number $(\mathrm{F})$ in the outer parenchymal cell layers of the lemma and palea of the wild-type and bsg1-1 $(n=10)$. All data are given as means \pm SD. The $P$-values were generated by Student's $t$-tests (in color in the electronic version).

division by regulating downstream cell-cycle genes to control grain shape and size.

Previous studies have reported that reduced expression of $G W 2$ increased grain width, whereas overexpression of $G W 2$ decreased grain size [5], and higher expression of GS5 was correlated with larger grain size owing to an increase of cell number in glumes [11]. We analyzed the transcript levels of GW2 and GS5 in the hulls of bsgl-1 and the wild-type, and found that $G W 2$ expression was significantly upregulated in 8-cm young panicles of bsgl-1 compared with the wild-type, whereas $G S 5$ expression changed only slightly in young panicles (Figure 6B). These observations suggested that BSG1 might be involved in the regulation pathway of GW2.

\subsection{BSG1 regulates genes in flower development}

It has been established that MADS-box genes play critical roles in the specification of floral organ identity in plants, 


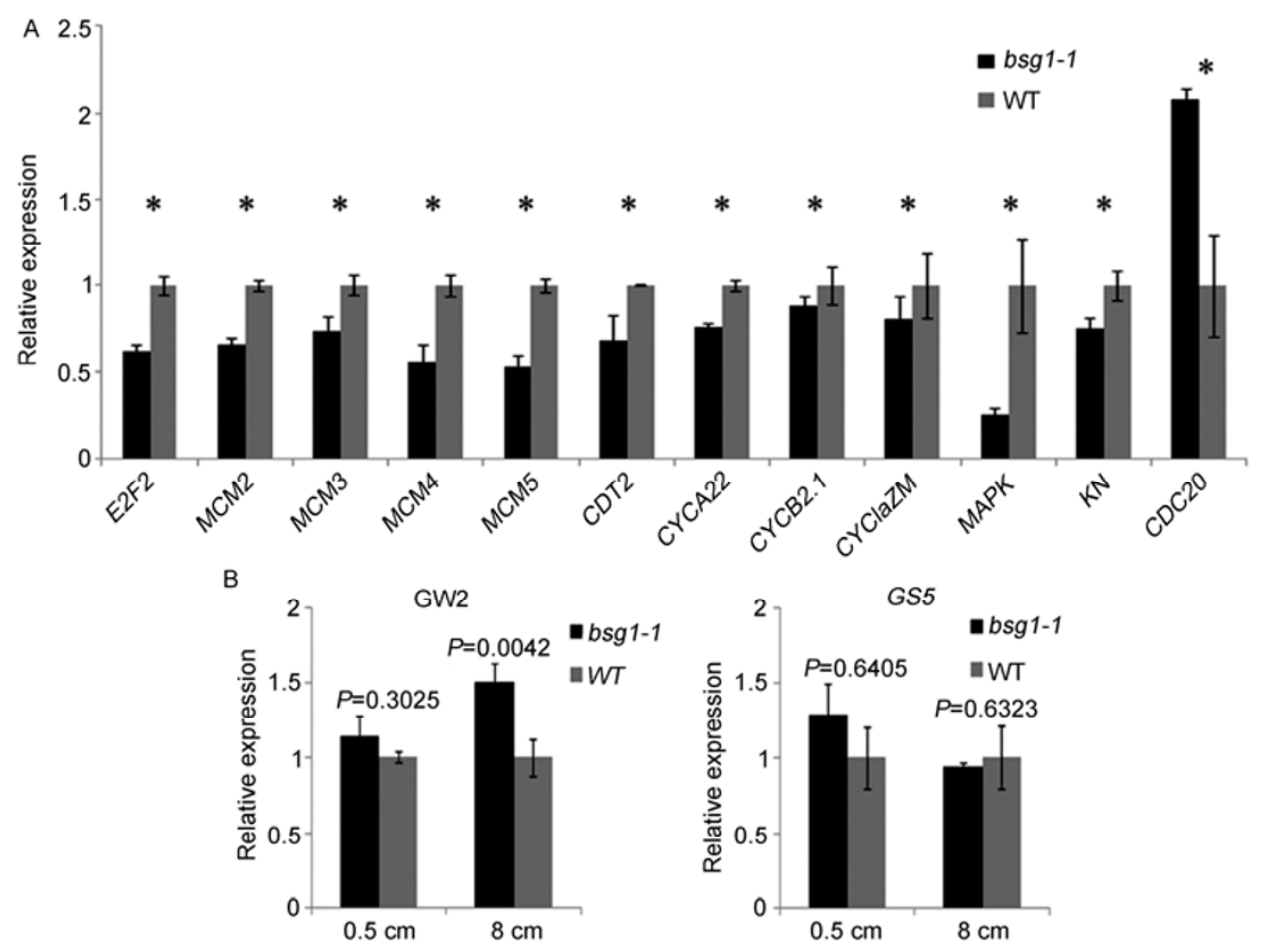

Figure 6 Expression levels of putative genes involved in cell division. A, Relative expression levels of putative rice cell cycle-related genes in wild-type and $b s g 1-1$ hulls. Asterisks indicate $P<0.05$. B, The different expression levels of $G W 2$ and $G S 5$ in young panicles (inflorescence length $0.5 \mathrm{~cm}$ and $8 \mathrm{~cm}$ ) of the wild-type and $b s g 1-1$. All data were determined from three replicates. All $P$-values are based on two-tailed $t$-tests.

including rice [33]. Thus, we assayed the expression levels of MADS-box genes involving in rice lemma/palea and lodicule development. The genes that showed significant differences in expression levels between $b$ sg $1-1$ and the WT are shown in Figure 7. The expression levels of E-class genes OsMADS1 and OsMADS6 were only down-regulated in $0.5-\mathrm{cm}$ young panicles, while OsMADS16 was downregulated in both $0.5-\mathrm{cm}$ and $8-\mathrm{cm}$ panicles (Figure 7). The transcript level of OsMADS55 was increased in $0.5-\mathrm{cm}$ young panicles but reduced in 8-cm panicles of the $b s \mathrm{gl}-1$

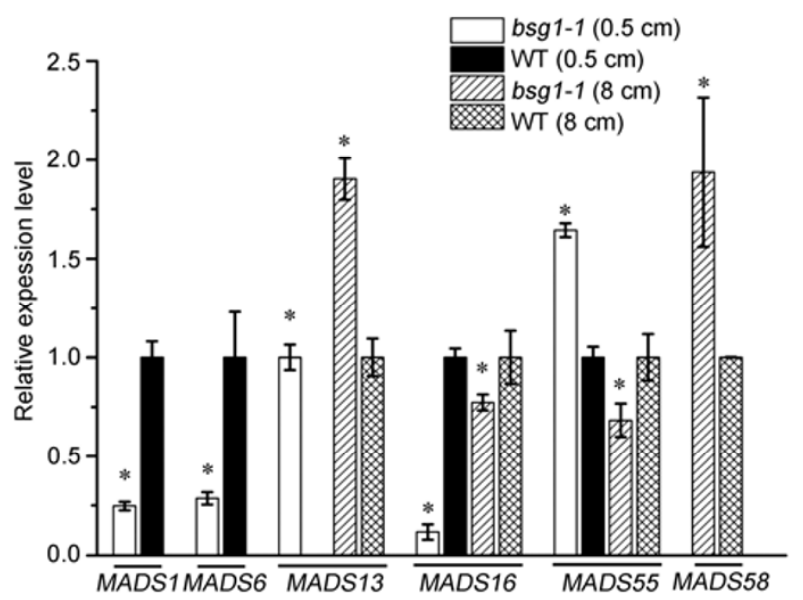

Figure 7 Real-time PCR analysis of MADS-box gene expression in young panicles (inflorescence length $0.5 \mathrm{~cm}$ and $8 \mathrm{~cm}$ ) of the wild-type and bsg1-1. ACTIN was used as a control. Asterisks indicate $P<0.05$. mutant (Figure 7). Moreover, we found that expression of the C-class gene OsMADS58 and the D-class gene Os$M A D S 13$ was up-regulated in $8-\mathrm{cm}$ panicles, and $O s$ $M A D S 13$ was also greatly up-regulated in $0.5-\mathrm{cm}$ panicles (Figure 7). These results implied that $B S G 1$ was also involved in floral organ formation and development.

\section{Discussion}

The shape and size of rice grains is controlled by cell division and differentiation, as well as by grain filling. The THI gene ( $B S G 1$ in our study) was previously reported to function in the control of rice seed shape and size [31]. However, it was unknown how THI influences the shape and size of the hull. The bsgl mutants developed small grains with reduced weight and thickness (Figure 1). Consistent with the grain phenotypes, histological observation showed that bsg 1-1 exhibited fewer and smaller cells in the outer parenchyma cell layers of the lemma and palea compared with wild-type plants. Moreover, gene expression assays indicated that most of the positive regulatory genes predicated to be involved in cell cycle control were down-regulated in the bsg1-1 mutant. Therefore, BSG1 most likely functions as a positive upstream modulator of cell division during rice floral organ development. Supporting this function, BSG1 was most strongly expressed in the young panicles (Figure 4D and $\mathrm{E})$. 
A database search indicated that there are 10 genes that encode proteins with DUF640 domains in both rice and Arabidopsis [34] (Figure 8A and B). LIGHT-DEPENDENT SHORT HYPOCOTYLS1 (LSH1), which mediates light regulation of seedling development via phytochrome, is the only DUF640-domain gene functionally elaborated in Arabidopsis [35]. In rice, the homeotic gene LONG STERILE LEMMA $(G l)$ was shown to be involved in transcriptional regulation and specification of sterile lemma identity [34]. LSH1 and G1 are both localized in the nucleus, similar to BSG1. In addition, we found that BSG1 has transcriptional activation ability using a yeast-two-hybrid assay (Figure S1).
Thus, it is likely that BSG1 functions at a specific regulatory node controlling cell division and expansion in the rice grain hull. Further studies will be required to clarify the underlying mechanisms by identifying the BSG1-interacting protein(s).

OsMADS55 functions as a negative regulator of brassinosteroid responses and stem elongation and senescence processes [36]. The expression level change of OsMADS55 in bsgl suggests the possible implication of BSG1 in Os$M A D S 55$-mediated inflorescence elongation and floral organ augmentation. Previous studies have shown that $O s$ MADS58 functions in the establishment of floral meristem
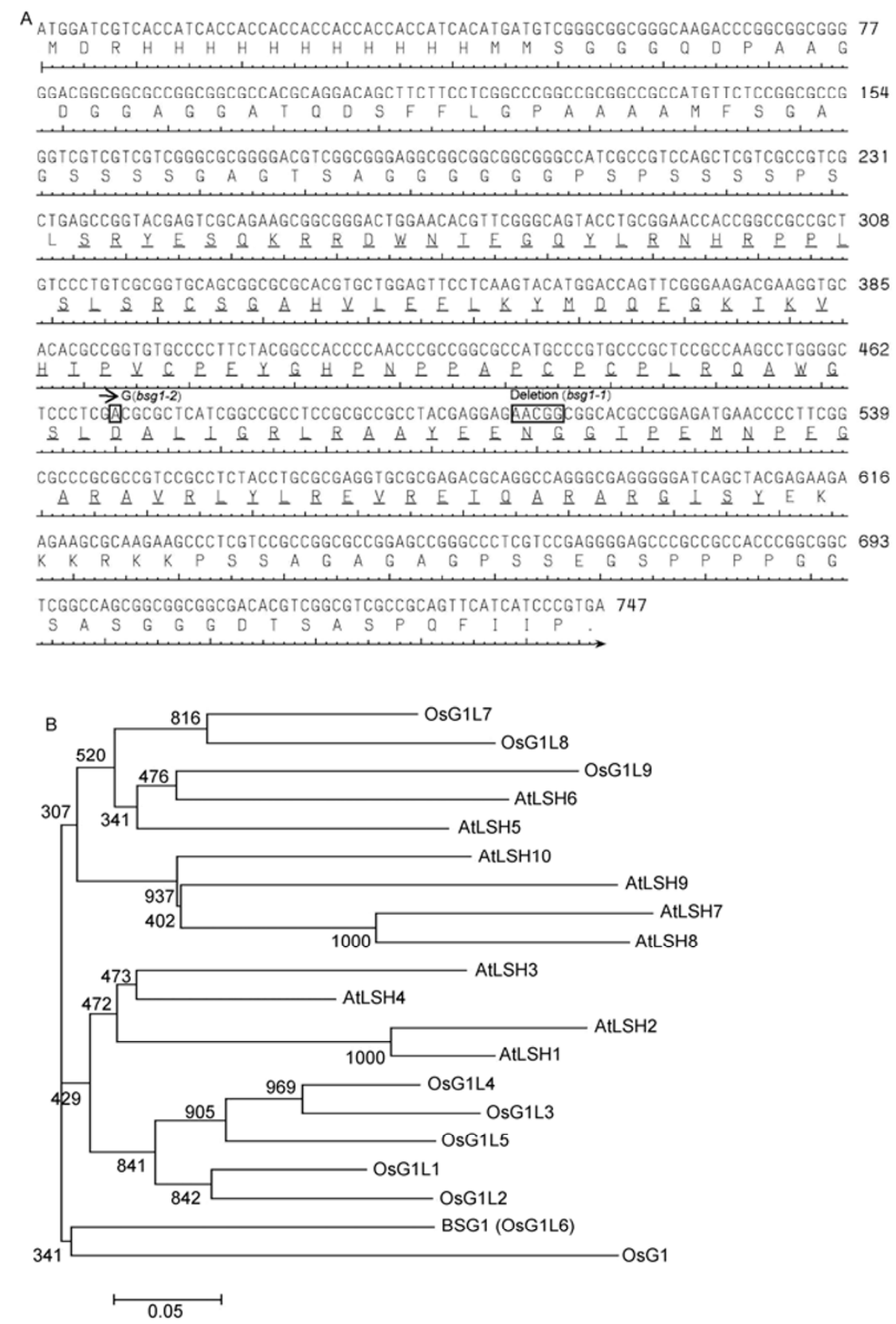

Figure 8 Phylogenetic tree of proteins containing the DUF640 domain. A, Protein and cDNA sequences of BSG1. Dashed lines indicate the DUF640 domain. Black boxes indicate the mutation sites in the bsg1 mutants. B, Phylogenetic tree of DUF-640 proteins in rice and Arabidopsis. The tree was constructed by the neighbor-joining method. Numbers indicate bootstrap values. 
determinacy and carpel development, while OsMADS13 is involved in ovule specification $[37,38]$. Thus, we speculate that $B S G 1$ might also participate in the regulation pathways of carpel and ovule development besides those of lem$\mathrm{ma}$ /palea and lodicule development. However, detailed expression pattern changes in different floral organs need to be analyzed and the mechanism of floral homeotic gene regulation by $B S G 1$ remains to be elucidated.

We thank Gao XiaoYan and Gao XiaoShu for assistance with scanning electron microscopy and confocal laser microscopy. This work was supported by grants from the Chinese Academy of Sciences (KSCX2-EW-N-01) and the National Natural Science Foundation of China (31100142, 31071207).

1 Xue W, Xing Y, Weng X, et al. Natural variation in Ghd7 is an important regulator of heading date and yield potential in rice. Nat Genet, 2008, 40: 761-767

2 Li S, Qian Q, Fu Z, et al. Short panicle1 encodes a putative PTR family transporter and determines rice panicle size. Plant J, 2009, 58: 592-605

3 Jiao Y, Wang Y, Xue D, et al. Regulation of OsSPL14 by OsmiR156 defines ideal plant architecture in rice. Nat Genet, 2010, 42: 541-544

4 Fan C, Xing Y, Mao H, et al. GS3, a major QTL for grain length and weight and minor QTL for grain width and thickness in rice, encodes a putative transmembrane protein. Theor Appl Genet, 2006, 112: 1164-1171

5 Song X J, Huang W, Shi M, et al. A QTL for rice grain width and weight encodes a previously unknown RING-type E3 ubiquitin ligase. Nat Genet, 2007, 39: 623-630

6 Shomura A, Izawa T, Ebana K, et al. Deletion in a gene associated with grain size increased yields during rice domestication. Nat Genet, 2008, 40: 1023-1028

7 Weng J, Gu S, Wan X, et al. Isolation and initial characterization of $G W 5$, a major QTL associated with rice grain width and weight. Cell Res, 2008, 18: 1199-1209

8 Wang E, Wang J, Zhu X, et al. Control of rice grain-filling and yield by a gene with a potential signature of domestication. Nat Genet, 2008, 40: 1370-1374

9 Ashikari M, Sakakibara H, Lin S, et al. Cytokinin oxidase regulates rice grain production. Science, 2005, 309: 741-745

10 Miura K, Ikeda M, Matsubara A, et al. OsSPL14 promotes panicle branching and higher grain productivity in rice. Nat Genet, 2010, 42: 545-549

11 Li Y, Fan C, Xing Y, et al. Natural variation in GS5 plays an important role in regulating grain size and yield in rice. Nat Genet, 2011, 43: 1266-1269

12 Mao H, Sun S, Yao J, et al. Linking differential domain functions of the GS3 protein to natural variation of grain size in rice. Proc Natl Acad Sci USA, 2010, 107: 19579-19584

13 Jeon J S, Jang S, Lee S, et al. leafy hull sterile1 is a homeotic mutation in a rice MADS box gene affecting rice flower development. Plant Cell, 2000, 12: 871-884

14 Yuan Z, Gao S, Xue D W, et al. RETARDED PALEA1 controls palea development and floral zygomorphy in rice. Plant Physiol, 2009, 149: 235-244

15 Jin Y, Luo Q, Tong H, et al. An AT-hook gene is required for palea formation and floral organ number control in rice. Dev Biol, 2011, 359: $277-288$

16 Horigome A, Nagasawa N, Ikeda K, et al.. Rice OPEN BEAK is a negative regulator of class 1 knox genes and a positive regulator of class B floral homeotic gene. Plant J, 2009, 58: 724-736

17 Inze D, de Veylder L. Cell cycle regulation in plant development. Annu Rev Genet, 2006, 40: 77-105

18 De Veylder L, Beeckman T, Inze D. The ins and outs of the plant cell cycle. Nat Rev Mol Cell Biol, 2007, 8: 655-665

19 Dewitte W, Murray J A. The plant cell cycle. Annu Rev Plant Biol, 2003, 54: 235-264

20 Magyar Z, Meszaros T, Miskolczi P, et al. Cell cycle phase specificity of putative cyclin-dependent kinase variants in synchronized alfalfa cells. Plant Cell, 1997, 9: 223-235

21 Sorrell D A, Menges M, Healy J M, et al. Cell cycle regulation of cyclin-dependent kinases in tobacco cultivar Bright Yellow-2 cells. Plant Physiol, 2001, 126: 1214-1223

22 Breyne P, Dreesen R, Vandepoele K, et al. Transcriptome analysis during cell division in plants. Proc Natl Acad Sci USA, 2002, 99: 14825-14830

23 Corellou F, Camasses A, Ligat L, et al. Atypical regulation of a green lineage-specific B-type cyclin-dependent kinase. Plant Physiol, 2005, 138: 1627-1636

24 Fobert P R, Gaudin V, Lunness P, et al. Distinct classes of cdc2-related genes are differentially expressed during the cell division cycle in plants. Plant Cell, 1996, 8: 1465-1476

25 Fabian-Marwedel T, Umeda M, Sauter M. The rice cyclin-dependent kinase-activating kinase R2 regulates S-phase progression. Plant Cell, 2002, 14: 197-210

26 Umeda M, Umeda-Hara C, Uchimiya H. A cyclin-dependent kinaseactivating kinase regulates differentiation of root initial cells in Arabidopsis. Proc Natl Acad Sci USA, 2000, 97: 13396-13400

27 Morgan D O. Cyclin-dependent kinases: engines, clocks, and microprocessors. Annu Rev Cell Dev Biol, 1997, 13: 261-291

28 De Veylder L, Beeckman T, Beemster G T, et al. Functional analysis of cyclin-dependent kinase inhibitors of Arabidopsis. Plant Cell, 2001, 13: $1653-1668$

29 Hiei Y, Ohta S, Komari T, et al. Efficient transformation of rice (Oryza sativa L.) mediated by Agrobacterium and sequence analysis of the boundaries of the T-DNA. Plant J, 1994, 6: 271-282

30 Livak K J, Schmittgen T D. Analysis of relative gene expression data using real-time quantitative PCR and the 2(-Delta Delta C(T)) Method. Methods, 2001, 25: 402-408

31 Li X, Sun L, Tan L, et al. TH1, a DUF640 domain-like gene controls lemma and palea development in rice. Plant Mol Biol, 2012, 78: 351-359

32 Iwata E, Ikeda $\mathrm{S}$, Matsunaga $\mathrm{S}$, et al. GIGAS CELL1, a novel negative regulator of the anaphase-promoting complex/cyclosome, is required for proper mitotic progression and cell fate determination in Arabidopsis. Plant Cell, 2011, 23: 4382-4393

33 Yamaguchi $\mathrm{T}$, Hirano $\mathrm{H} \mathrm{Y}$. Function and diversification of MADS-box genes in rice. Sci World J, 2006, 6: 1923-1932

34 Yoshida A, Suzaki T, Tanaka W, et al. The homeotic gene long sterile lemma $(G 1)$ specifies sterile lemma identity in the rice spikelet. Proc Natl Acad Sci USA, 2009, 106: 20103-20108

35 Zhao L, Nakazawa M, Takase T, et al. Overexpression of LSH1, a member of an uncharacterised gene family, causes enhanced light regulation of seedling development. Plant J, 2004, 37: 694-706

36 Lee S, Choi S C, An G. Rice SVP-group MADS-box proteins, OsMADS22 and OsMADS55, are negative regulators of brassinosteroid responses. Plant J, 2008, 54: 93-105

37 Yamaguchi T, Lee D Y, Miyao A, et al. Functional diversification of the two C-class MADS box genes OSMADS3 and OSMADS58 in Oryza sativa. Plant Cell, 2006, 18: 15-28

$38 \mathrm{Li} \mathrm{H}$, Liang W, Hu Y, et al. Rice MADS6 interacts with the floral homeotic genes SUPERWOMAN1, MADS3, MADS58, MADS13, and DROOPING LEAF in specifying floral organ identities and meristem fate. Plant Cell, 2011, 23: 2536-2552

Open Access This article is distributed under the terms of the Creative Commons Attribution License which permits any use, distribution, and reproduction in any medium, provided the original author(s) and source are credited. 


\section{Supporting Information}

Table S1 Primers used in this study

Figure S1 Subcellular localization of BSG1 and transcriptional activation assay. A, BSG1 was localized in the nucleus. B, Transcriptional activation analysis of BSG1 in a yeast-two-hybrid system.

The supporting information is available online at life.scichina.com and www.springerlink.com. The supporting materials are published as submitted, without typesetting or editing. The responsibility for scientific accuracy and content remains entirely with the authors. 